\title{
DIMENSION SEQUENCES FOR COMMUTATIVE RINGS
}

\author{
BY JIMMY T. ARNOLD AND ROBERT GILMER ${ }^{1}$ \\ Communicated by Oscar Goldman, September 19, 1972
}

Let $R$ be a commutative ring with identity of finite (Krull) dimension $n_{0}$, and for each positive integer $k$, let $n_{k}$ be the dimension of the polynomial ring $R^{(k)}=R\left[X_{1}, \ldots, X_{k}\right]$ in $k$ indeterminates over $R$. The sequence $\left\{n_{i}\right\}_{i=0}^{\infty}$ is called the dimension sequence for $R$, and the sequence $\left\{d_{i}\right\}_{i=1}^{\infty}$, where $d_{i}=n_{i}-n_{i-1}$ for each $i$, is called the difference sequence for $R$. We are concerned with a determination of those sequences of nonnegative integers that can be realized as the dimension sequence of a ring.

Several restrictions on the sequences $\left\{n_{i}\right\}$ and $\left\{d_{i}\right\}$ are known. For example, $n_{k}+1 \leqq n_{k+1} \leqq 2 n_{k}+1$ for each positive integer $k$ [5], and $n_{0}+k \leqq n_{k} \leqq\left(n_{0}+1\right)(k+1)-1$ [4]. In particular, the only dimension sequence for a zero-dimensional ring is $0,1,2, \ldots$. Jaffard in [4] proved that the difference sequence for $R$ is eventually a constant less than or equal to $n_{0}+1$-that is, there is a positive integer $k$ such that $n_{0}+1 \geqq$ $d_{k}=d_{k+1}=\cdots$. Moreover, if $R$ is an integral domain, then both the integer $k$ and the value $d_{k}$ relate to the valuative dimension $\operatorname{dim}_{v} R$, defined to be $\sup \{$ rank $V \mid V$ is a valuation overring of $R\}$. Jaffard proved that the eventual value of the difference sequence for $R$ is 1 if and only if $R$ has finite valuative dimension. In fact, if $\operatorname{dim}_{v} R=k<\infty$, then Jaffard proved that $d_{i}=1$ for $i \geqq k+1$; in [1], Arnold improved Jaffard's bound by 1 to $i \geqq k$, and this bound, in turn, cannot be improved in the general case.

We are able to prove that the restrictions mentioned in the previous paragraph are all that are necessary in order that an increasing sequence $\left\{m_{i}\right\}_{i=0}^{\infty}$ of positive integers with nonincreasing difference sequence $\left\{m_{i}-m_{i-1}\right\}_{i=1}^{\infty}$ should be the dimension sequence of an integral domain. It is known, however, that the difference sequence for an integral domain need not be nonincreasing, and hence we require additional restrictions to solve the general problem.

We denote by $\mathscr{S}$ the set of sequences $\left\{m_{i}\right\}_{i=0}^{\infty}$ of positive integers such that the corresponding difference sequence $\left\{t_{i}\right\}_{i=1}^{\infty}$, where $t_{i}=m_{i}-m_{i-1}$, satisfies the following conditions:

(1) $m_{0}+1 \geqq t_{1} \geqq t_{2} \geqq \cdots$.

(2) There is a positive integer $k$ such that $1 \leqq t_{k}=t_{k+1}=t_{k+2}=\cdots$.

AMS(MOS) subject classifications (1970). Primary 13C05, 13F20, 13J05; Secondary 13G05, 13G15, 13E05, 13E10.

Key words and phrases. Polynomial ring, dimension, chains of prime ideals, $D+M$ construction.

${ }^{1}$ Research supported by NSF Grant GP-33027x.

Copyright (C) American Mathematical Society 1973 
THEOREM 1. If $F$ is a field, if $\left\{X_{i}\right\}_{i=0}^{\infty}$ is a set of indeterminates over $F$, and if $s \in \mathscr{S}$, then there is an integrally closed quasi-local domain $D$ such that $D$ has quotient field $F\left(\left\{X_{i}\right\}_{0}^{\infty}\right)$, the set of prime ideals of $D$ is linearly ordered by inclusion, and $D$ has dimension sequence $s$.

The proof of Theorem 1 uses induction on $s_{0}$, the first term in the sequence $s$, and a theorem of Bastida and Gilmer in [2] that relates the dimension sequence for the domain $D$ to that of the domain $D_{1}=D+M$, where $D$ is a subring of the field $K$ and $M$ is the maximal ideal of a valuation ring of the form $K+M$. If $s_{0}=0$, Theorem 1 is trivial; otherwise, we modify the sequence $s=\left\{s_{i}\right\}_{0}^{\infty}$ as follows. If the difference sequence $\left\{d_{i}\right\}_{1}^{\infty}$ for $s$ is eventually 1 , we let $v$ be the first positive integer for which $d_{v}=1$; otherwise, we set $v=\infty$. We define a sequence $s^{\prime}=\left\{s_{i}^{\prime}\right\}_{i=0}^{\infty}$ by $s_{0}^{\prime}=s_{0}-1, s_{i}^{\prime}=s_{i-1}^{\prime}+d_{i}^{\prime}$, where $d_{i}^{\prime}=d_{i}$ if $d_{i}=1$ and $d_{i}^{\prime}=d_{i}-1$ if $d_{i}>1$. If $A \cup B$ is a partition of the set of positive integers in such a way that $A$ is infinite and $B$ has cardinality $v-1$, then we can assume that Theorem 1 is true for the sequence $s^{\prime}$ and the field $F\left(\left\{X_{i}\right\}_{i \in A}\right)$, and then results of [2] allows us to extend to the sequence $s$, using the valuation ring $V=F\left(\left\{X_{i}\right\}_{1}^{\infty}\right)\left[X_{0}\right]_{\left(X_{0}\right)}=F\left(\left\{X_{i}\right\}_{1}^{\infty}\right)+X_{0} V$ on $F\left(\left\{X_{i}\right\}_{0}^{\infty}\right)$.

For $s_{1}, s_{2}, \ldots, s_{r}$ in $\mathscr{S}$, with $s_{i}=\left\{s_{j}^{(i)}\right\}_{j=0}^{\infty}$, we define $\sup \left\{s_{1}, \ldots, s_{r}\right\}$ to be the sequence $\left\{\left\{\max s_{j}^{(i)}\right\}_{i=1}^{r}\right\}_{j=0}^{\infty}$, and we let

$$
\mathscr{D}=\left\{\sup \left\{s_{1}, \ldots, s_{r}\right\} \mid s_{1}, s_{2}, \ldots, s_{r} \in \mathscr{S}\right\} .
$$

There are two primary results concerning the set $\mathscr{D}$.

THEOREM 2. If $F$ is a field, if $\left\{X_{i}\right\}_{0}^{\infty}$ is a set of indeterminates over $F$, and if $s \in \mathscr{D}$, then there is an integrally closed semi-quasi-local domain $D$ with quotient field $F\left(\left\{X_{i}\right\}_{0}^{\infty}\right)$ such that $s$ is the dimension sequence for $D$.

THEOREM 3. If $R$ is a commutative ring with identity, then the dimension sequence for $R$ is in $\mathscr{D}$. In particular, the dimension sequence for $R$ is the dimension sequence of a semi-quasi-local integral domain.

Theorems 2 and 3 and their proofs provide a satisfactory answer to the question as to what sequences are dimension sequences. The proof of Theorem 2 depends very heavily upon a construction of Gilmer in [3]. This construction begins with a finite family $\left\{V_{i}\right\}_{i=1}^{n}$, of valuation rings on a field $L$, each of the form $K+M_{i}$, where $K$ is a subfield of $L$ and $M_{i}$ is the maximal ideal of $V_{i}$. The domain $D$ used in the proof of Theorem 2 is of the form $\bigcap_{i=1}^{n}\left(D_{i}+M_{i}\right)$, where $D_{i}$ is a subring of $K$ for each $i$ between 1 and $n$. Because of the technical nature of the proof of Theorem 2, no outline of the proof will be given here. We do remark, however, that an extension of the results of [3] concerning the structure of $\bigcap_{1}^{n}\left(D_{i}+M_{i}\right)$ is necessary for us to obtain the proof of Theorem 2; in particular, the result that each $D_{i}+M_{i}$ is a localization of $\bigcap_{1}^{n}\left(D_{i}+M_{i}\right)$ is needed. 
In order to prove Theorem 3, we require an analysis of the way lengths of chains of proper prime ideals of $R^{(m)}$ behave under intersection with $R^{(k)}$, for $k \leqq m$. In particular, we make heavy use of the so-called Special Chain Theorem of Jaffard [4]. The main consequence of this theorem that we need is the result that $\operatorname{rank} P=\operatorname{rank}(P \cap R)^{(m)}+\operatorname{rank} P /(P \cap R)^{(m)}$ for each proper prime ideal $P$ of $R^{(m)}$.

Let $\mathscr{C}$ be a chain of prime ideals of $R^{(m)}$, and for $0 \leqq i \leqq m$, let $\mathscr{C}_{i}$ be the intersection of $\mathscr{C}$ with $R^{(i)}$. We say that $\mathscr{C}$ is a $k$-chain if the following conditions are satisfied.

(1) $\mathscr{L}(\mathscr{C})=k$.

(2) $\mathscr{L}\left(\mathscr{C}_{i}\right)-\mathscr{L}\left(\mathscr{C}_{i-1}\right) \leqq \mathscr{L}\left(\mathscr{C}_{0}+1\right)$ for $1 \leqq i \leqq m$.

(3) $\mathscr{L}\left(\mathscr{C}_{i+1}\right)-\mathscr{L}\left(\mathscr{C}_{i}\right) \leqq \mathscr{L}\left(\mathscr{C}_{i}\right)-\mathscr{L}\left(\mathscr{C}_{i-1}\right)$ for $1 \leqq i \leqq m-1$.

Moreover, if the condition

(4) $1 \leqq \mathscr{L}\left(\mathscr{C}_{i}\right)-\mathscr{L}\left(\mathscr{C}_{i-1}\right)$ for $1 \leqq i \leqq m$

is also satisfied, then we call $\mathscr{C}$ a $k^{*}$-chain. The following two results are essential to the proof of Theorem 3 .

THEOREM 4. If $P$ is a proper prime ideal of $R^{(m)}$ of rank $k$, then $P$ is the terminal element of a $k$-chain of prime ideals of $R^{(m)}$.

THEOREM 5. If $M$ is a maximal ideal of $R^{(m)}$ of rank $k$, then $M$ is the terminal element of $a k^{*}$-chain of prime ideals of $R^{(m)}$.

Details and proofs of this work will appear elsewhere.

\section{REFERENCES}

1. J. T. Arnold, On the dimension theory of overrings of an integral domain, Trans. Amer. Math. Soc. 138 (1969), 313-326. MR 39 \# 188.

2. E. Bastida and R. Gilmer, Overrings and divisorial ideals of domains of the form $D+M$, Michigan Math. J. (to appear).

3. R. Gilmer, Two constructions of Prüfer domains, J. Reine Angew. Math. 239/240 (1969), 153-162. MR 41 \# 1710.

4. P. Jaffard, Théorie de la dimension dans les anneaux de polynomes, Mémor. Sci. Math., fasc. 146, Gauthier-Villars, Paris, 1960. MR 22 \#8038.

5. A. Seidenberg, $A$ note on the dimension theory of rings, Pacific J. Math. 3 (1953), 505-512. MR 14, 941 .

Department of Mathematics, Virginia Polytechnic Institute and State University, BLACKSBURG, Virginia 24061.

Department of Mathematics, Florida State University, Tallahassee, Florida 32306. 